\title{
Difunctionalization of Acrylamides through C-H Oxidative Radical Coupling: New Approaches to Oxindoles
}

\section{Ren-Jie Song \\ Yu Liu \\ Ye-Xiang Xie \\ Jin-Heng Li*}

State Key Laboratory of Chemo/Biosensing and Chemometrics, College of Chemistry and Chemical Engineering, Hunan University, Changsha 410082, P. R. of China

jhli@hnu.edu.cn

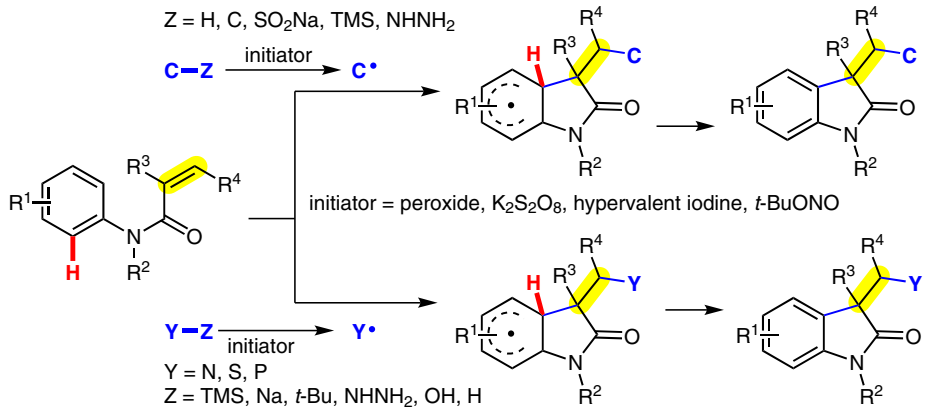

Received: 31.12.2014

Accepted after revision: 28.01.2015

Published online: 31.03 .2015

DOI: 10.1055/s-0034-1379903; Art ID: ss-2014-e0784-r

Abstract Oxindoles are an important class of heterocycles with unique biological activity that are found prevalently in numerous natural products and biologically active compounds. For these reasons, much attention has been given to the development of efficient methods for the preparation of such compounds. Traditionally, the practical approaches for the synthesis of oxindoles include the condensation of anilines with carbonyl compounds, such as diethyl ketomalonate, oxalyl chloride, or chloral hydrate, mediated by strong acids or bases. Recently, a step- and atom-economic $\mathrm{C}-\mathrm{H}$ activation strategy was illustrated to access oxindoles through the difunctionalization of activated alkenes for these purposes. However, many of these transformations suffer from the cost of the transition-metal catalytic systems and/or limited substrate scope. In this review, we describe the recent studies of the difunctionalization of activated alkenes for the synthesis of diverse functionalized oxindoles that involves $\mathrm{C}-\mathrm{H}$ oxidative radical coupling in the presence of an oxidant. These transformations are initiated either by the carbon radical resulting from the split of the carbon-hydrogen bond (1,2-dicarbofunctionalization) or by the carbon or heteroatom radical arising from the cleavage of the carbon-heteroatom (1,2-dicarbofunctionalization) or heteroatom-heteroatom bond (1,2-carboheterofunctionalization). Importantly, these $\mathrm{C}-\mathrm{H}$ oxidative radical coupling transformations are generally performed with readily available oxidants and/or inexpensive iron or copper catalysts under neutral reaction conditions.

1 Introduction

2 Synthesis of Oxindoles via 1,2-Dicarbofunctionalization of Alkenes

2.1 1,2-Alkylarylation

2.2 1,2-Aryltrifluoromethylation

2.3 1,2-Carbonylarylation

3 Synthesis of Oxindoles via 1,2-Carboheterofunctionalization of Alkenes

3.1 1,2-Azidoarylation or 1,2-Arylnitration

3.2 1,2-Arylsulfonylation

3.2 1,2-Arylphosphorylation

4 Conclusion

Key words oxindoles, difunctionalization, $\mathrm{C}-\mathrm{H}$ oxidative radical coupling, radical reaction, alkenes

\section{Introduction}

Oxindoles, an important class of heterocycles with a wide range of biological properties, are a key structural motif in numerous natural products and biologically active ant synthetic intermediates that have elicited considerable synthetic interest principally because of their important applications in asymmetric synthesis, library design and drug discovery. ${ }^{2}$ As a result, efficient methods for oxindole synthesis have been the subject of extensive studies. Among these methods, the recently developed transitionmetal-catalyzed or metal-free oxidative difunctionalization<smiles>COc1ccc2c(c1)[C@@]1(CCN(C)C1)C(=O)N2</smiles>

horsfiline<smiles></smiles>

alstonisine

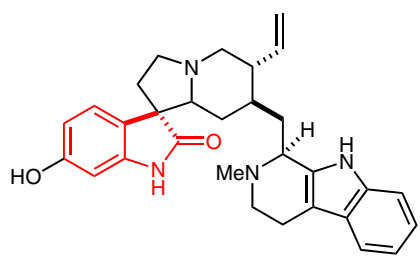

strychnofoline<smiles>COc1ccccc1C1(OC(C)=O)C(=O)N([O+])c2ccc(Cl)cc21</smiles><smiles>CCN(CCl)CCN1C(=O)[C@@](O)(c2ccccc2Cl)c2c1cc(C(N)=O)cc2C(F)(F)F</smiles>

SM-130686<smiles>COc1ccc2c(c1)NC(=O)C21C[C@H]2C(=O)N3CCC[C@H]3C(=O)N2C1=CC(C)C</smiles>

spirotryprostain A
Figure 1 Examples of important oxindoles compounds (Figure 1). ${ }^{1}$ Additionally, oxindoles are import- 
of activated alkenes is a particularly fascinating approach to building diversely functionalized oxindoles, owing to its high step- and atom-economy, and easy incorporation of many functional groups (e.g., cyano, carbonyl, hydroxyl, phosphoryl, trifluoromethyl, azidyl, and nitro) into the oxindole framework.

The difunctionalizations of alkenes have been significantly developed and have already played vital roles in organic syntheses. ${ }^{3}$ In particular, the applications of difunctionalization of alkenes for the synthesis of important bio- active heterocyclic compounds has proven to be a powerful alternative to the traditional approaches. Recent studies have illustrated that these transformations, such as arylalkylation, aminooxygenation, diamination, and dioxygenation of unsaturated hydrocarbons, could be used efficiently to allow the formation of diverse chemical bonds. In 1999, the Grigg research group reported a palladium-catalyzed difunctionalization of alkenes with aryl iodides and carbon monoxide through an intramolecular Heck-carbonylation reaction for the synthesis of oxindoles and their de-



Yu Liu was born in Hunan, P. R. of China, in 1985. He received his B.S. (in 2008) and M.S. degrees (in 2011) from College of Chemistry and Chemical Engi- neering at Hunan Normal University. He is currently studying towards a Ph.D. at Hunan University under the supervision of Professor Jin-Heng Li. His cur- rent research interests are focused on 1,n-enyne oxidative radical cyclization reactions.
Ye-Xiang Xie was born in Hunan, P. R. of China, in 1975. She received her B.S. degree in 1999 from Hunan Agricultural University and M.S. degree in 2007 from Hunan Normal University.
From 2010 to 2014, she undertook Ph.D. studies at Hunan University under the supervision of Professor Jian-Nan Xiang and Professor Jin-Heng Li. Currently, she is a lecturer at Hunan
University. Her research interests are focused on transitionmetal-catalyzed $\mathrm{C}-\mathrm{H}$ oxidative arylation reactions.
Jin-Heng Li was born in Hunan, P. R. of China, in 1971. He received his B.S. in 2006 from College of Chemistry and Chemical Engineering at Hunan Normal University. From 1997 to 2010 , he studied at Guangzhou Institute of Chemistry, Chinese Academy of Sciences, where he obtained his M.S. degree under the supervision of Professor
Huanfeng Jiang. After completing his Ph.D. studies at University of Science and Technology of China in 2013 under the supervision of Professor Huanfeng Jiang and Professor Ming-Cai Chen, he continued his studies as a postdoctoral fellow with Professor Dan Yang at the University of Hong Kong (P. R. of China). In 2002, he joined the faculty at Hunan Normal University as a professor. Since 2011 , he has worked as a professor in College of Chemistry and Chemical Engineering at Hunan University. His current research interests are focused on $\mathrm{C}-\mathrm{H}$ oxidative coupling reactions, cross-coupling reactions, and cycloaddition reactions. 
rivatives in good yield (Scheme 1). ${ }^{4}$ Following on these results, a similar version of palladium(0)-catalyzed alkene difunctionalization involving the intramolecular Heck coupling of $o$-haloanilines has been developed for accessing oxindoles and their derivatives. ${ }^{5}$

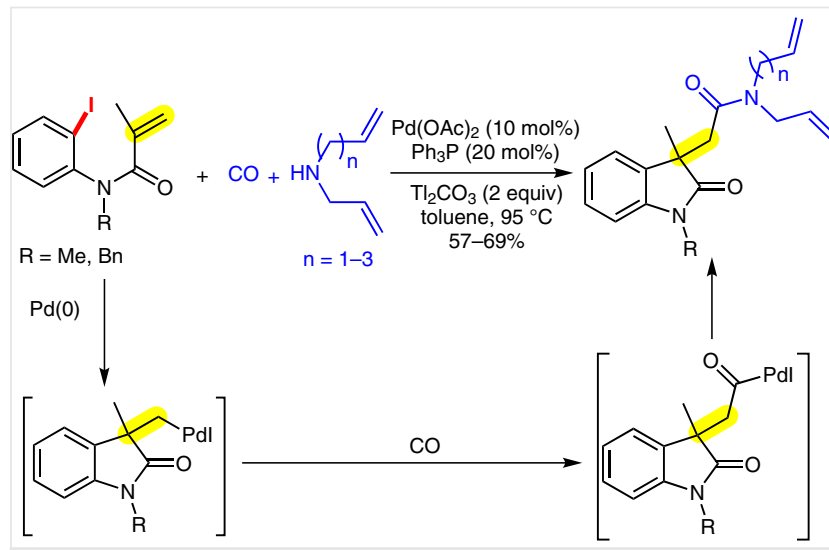

Scheme 1 Palladium-catalyzed domino intramolecular Heck-carbonylation reaction

In 2007, the Zhu research group expanded the palladium-catalyzed domino intramolecular Heck coupling protocol to assemble 3-substituted 3-cyanomethyl-2-oxindoles (Scheme 2), ${ }^{6}$ in which $\mathrm{K}_{4}\left[\mathrm{Fe}(\mathrm{CN})_{6}\right]$ was employed as a trapping agent for the $\pi$-alkylpalladium intermediate. The reaction was applicable to a wide range of $o$-haloaniline substrates with different electronic properties. In addition, a concise synthesis of physostigmine utilizing this key domino process for the construction of the core framework was accomplished. Importantly, an enantioselective version of this domino process was also examined. However, these transformations also suffer from the cost of the palladiumligand catalytic system and the requirement of expensive and unavailable $\boldsymbol{o}$-haloaniline substrates.

To make the palladium-catalyzed domino methods more useful, Neuville and Zhu and their co-workers discovered a new palladium(II)/(diacetoxy)iodobenzene catalytic system for use in the domino cyclization of ortho-unsubstituted anilines through the $\mathrm{C}\left(\mathrm{sp}^{2}\right)-\mathrm{H}$ oxidative Heck-type coupling (Scheme 3). ${ }^{7}$ This method represents a new palladium-catalyzed oxidative carboheterofunctionalization of ortho-unsubstituted anilines via $\mathrm{C}\left(\mathrm{sp}^{2}\right)-\mathrm{H}$ oxidative activation, and provides a facile and highly atom-economic access to oxindoles.

The Liu research group described new palladium-catalyzed oxidative difunctionalization reactions of alkenes with acetonitriles ${ }^{8 a}$ or trimethyl(trifluoromethyl)silane ${ }^{8 b}$ for the synthesis of functionalized oxindoles through $\mathrm{C}-\mathrm{H}$ oxidative activation under neutral conditions (Scheme 4).

The mechanism for the palladium-catalyzed oxidative 1,2-difunctionalization of alkenes with acetonitriles involving a palladium(II)/palladium(IV) catalytic cycle, outlined in



Scheme 2 Possible mechanism for the palladium-catalyzed domino intramolecular Heck-cyanation reaction.

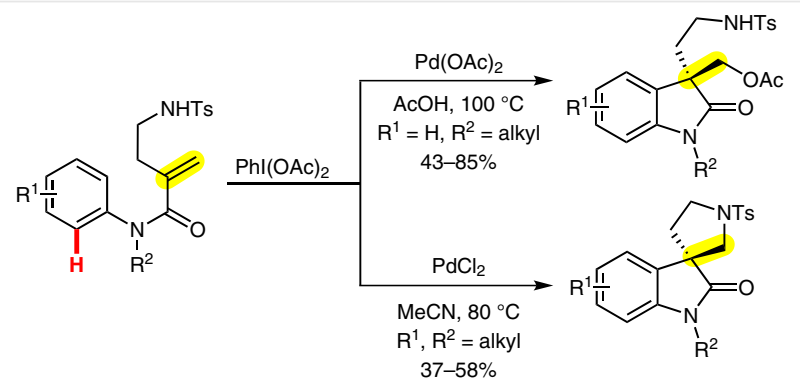

Scheme 3 Palladium-catalyzed oxidative 1,2-carboheterofunctionalization of alkenes

Scheme 5, was proposed on the basis of kinetic isotopic effect studies. The reaction is initiated by coordination of the olefin to palladium(II) (intermediate $\mathbf{A}$ ), followed by nucleophilic attack of the tethered arene to give the palladium complex $\mathbf{B}$. The $\mathrm{C}\left(\mathrm{sp}^{3}\right)-\mathrm{H}$ bond activation of the acetonitrile takes place in the presence of di(pivaloyloxy)iodobenzene and silver fluoride, thus generating the palladium(IV) complex $\mathbf{C}$. Finally, reductive elimination of intermediate $\mathbf{C}$ affords the desired oxindole. The formation of the palladium complex $\mathbf{B}$ is supported by HRMS analysis.

These palladium-catalyzed oxidative difunctionalization transformations involving $\mathrm{C}-\mathrm{H}$ activation are more attractive because it extends the substrate scope to ortho-unsubstituted anilines and can introduce additional functional groups into the oxindole framework. However, the use of the highly expensive palladium-hypervalent iodine reagent systems still limits the applicability of the reaction. Thus, the development of new $\mathrm{C}-\mathrm{H}$ oxidative coupling strategies for alkene difunctionalization, especially using inexpensive metal catalysts or no metal at all, is desirable. 

<smiles>CC(=O)OC[C@@]1(CCC#N)C(=O)N(c2ccccc2)c2ccccc21</smiles><smiles>C[C@H](C#N)C[C@@]1(C)C(=O)N(C)c2ccccc21</smiles><smiles></smiles>

$82 \%$<smiles>CC1(CC(F)(F)F)C(=O)N2CCCc3cccc1c32</smiles>

$81 \%$

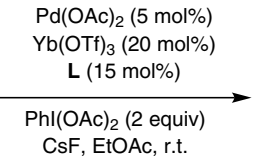

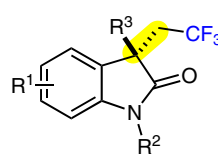<smiles>c1ccc2c(c1)C[C@H]1OC(CC3=N[C@@H]4c5ccccc5C[C@H]4O3)=N[C@H]21</smiles>

Scheme 5 Possible mechanism for the palladium-catalyzed oxidative alkylarylation of alkenes with acetonitriles

This review focuses on the construction of oxindoles by the difunctionalization of activated alkenes through $\mathrm{C}-\mathrm{H}$ oxidative radical coupling using inexpensive iron or copper catalysts as well as under metal-free conditions. It should be noted that the mechanisms of these alkene difunctionalization reactions through $\mathrm{C}-\mathrm{H}$ oxidative radical coupling are still unclear and remain to be studied. These transformations are classified, according to the difunctionalization process, into two types (Scheme 6): synthesis of oxindoles via 1,2-dicarbofunctionalization of alkenes (route a: the addition of carbon radicals across the carbon-carbon double bond, followed by arylation), and synthesis of oxindoles via 1,2-carboheterofunctionalization of alkenes (route $b$ : the addition of heteroatom and carbon radicals across the carbon-carbon double bond, followed by arylation; heteroatoms include N, S and P). The scope and limitations of these difunctionalization reactions are discussed, as well as their mechanisms.

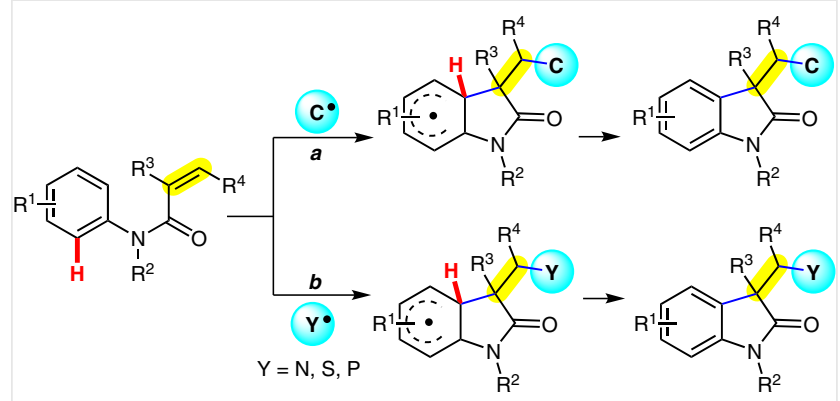

Scheme 6 Difunctionalization of activated alkenes through C-H oxidative radical coupling

\section{Synthesis of Oxindoles via 1,2-Dicarbo- functionalization of Alkenes}

It is well known that carbon-carbon bond-forming reactions are the most common processes in chemistry because they are important in the production of many useful chemicals such as pharmaceuticals and plastics. ${ }^{9}$ Among 
the numerous reported carbon-carbon bond-formation methods, those involving $\mathrm{C}-\mathrm{H}$ functionalization are particularly significant and remain challenging.

\subsection{1,2-Alkylarylation}

Encouraged by the results of the Neuville/Zhu and Liu research groups, $\mathrm{Li}$ and co-workers chose to use ethers compounds containing the activated $\mathrm{C}\left(\mathrm{sp}^{3}\right)-\mathrm{H}$ bond adjacent to the oxygen atom - in the presence of oxidants (often peroxides) to realize the 1,2-difunctionalization of alkenes (Scheme 7). ${ }^{10}$ In the presence of iron(III) chloride, tert-butyl hydroperoxide (TBHP) and 1,8-diazabicyclo[5.4.0] undec-7ene (DBU), a variety of activated alkenes reacted successfully with an aryl $\mathrm{C}\left(\mathrm{sp}^{2}\right)-\mathrm{H}$ bond and a $\mathrm{C}\left(\mathrm{sp}^{3}\right)-\mathrm{H}$ bond adjacent to a heteroatom, giving functionalized 3-(2-oxoethyl)indolin-2-ones in moderate to high yields. This oxidative 1,2-alkylarylation was catalyzed by the economical and environmentally benign metal iron, and was the first example of metal-catalyzed 1,2-difunctionalization of an alkene with an aryl $\mathrm{C}\left(\mathrm{sp}^{2}\right)-\mathrm{H}$ bond and a $\mathrm{C}\left(\mathrm{sp}^{3}\right)-\mathrm{H}$ bond adjacent to a heteroatom for the simultaneous formation of two carboncarbon bonds. Interestingly, the dual $\mathrm{C}-\mathrm{H}$ oxidative radical coupling protocol was applicable to thioethers and tertiary alkylamines as well.



selected products<smiles>CCOC(C)CC1(C)C(=O)N(C)c2ccccc21</smiles><smiles>CCCCCCCCCCCCC1(C)C(=O)N([18OH])c2ccccc21</smiles><smiles>CN1C(=O)C(C)(CC2COCCO2)c2ccccc21</smiles><smiles>CN1C(=O)C(C)(CC2CCCCS2)c2ccccc21</smiles><smiles>CN1C(=O)C(C)(CCN2CCCCC2)c2ccccc21</smiles>

$40 \%$

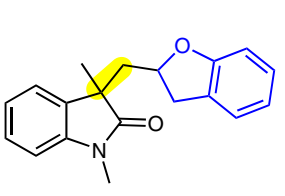

$51 \%$
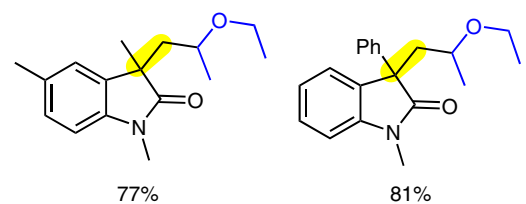

Scheme 7 Iron-catalyzed 1,2-alkylarylation of alkenes with the $C\left(\mathrm{sp}^{3}\right)$ $\mathrm{H}$ bonds adjacent to a heteroatom

Control experiments indicated that there was no kinetic isotope effect $\left(k_{H} / k_{D}=1.0\right)$ in the intramolecular or intermolecular experiments (Scheme 8). These observations im- plied that the iron-catalyzed alkene oxidative difunctionalization may proceed via either the SEAr mechanism or the free-radical mechanism. The results of the experiment with tetrahydrofuran and tetrahydrofuran- $d_{8}\left(k_{H} / k_{D}=1.0\right)$ supported the free-radical mechanism. To verify this mechanism, two radical inhibitors, TEMPO and 2,6-di-tert-butylphenol, were added to the difunctionalization reaction: a stoichiometric amount of radical inhibitor resulted in no conversion of amide; however, diethyl ether was transformed by TEMPO into 1-(1-ethoxyethoxy)-2,2,6,6-tetramethylpiperidine in $91 \%$ yield, suggesting that the reaction includes a diethyl ether radical.



Scheme 8 Control experiments

Consequently, Li and co-workers proposed the radical mechanism outlined in Scheme 9 for the iron-catalyzed 1,2-alkylarylation of alkenes with $\mathrm{C}\left(\mathrm{sp}^{3}\right)-\mathrm{H}$ bonds adjacent to a heteroatom. Initially, tert-butyl hydroperoxide is split by iron(II) into a tert-butoxy radical and a hydroxy-iron(III) species. In the presence of a tert-butoxy radical, diethyl ether, with its $\mathrm{C}\left(\mathrm{sp}^{3}\right)-\mathrm{H}$ bonds adjacent to oxygen, is readily transformed into alkyl radical intermediate $\mathbf{D}$. Addition of alkyl radical intermediate $\mathbf{D}$ to the carbon-carbon double bond of $\mathrm{N}$-arylmethacrylamide results in the formation of radical intermediate $\mathbf{E}$, which undergoes the intramolecular cyclization with the $\mathrm{C}\left(\mathrm{sp}^{2}\right)-\mathrm{H}$ bond in the $\mathrm{N}$-aryl ring to generate radical intermediate $\mathbf{F}$. Finally, hydrogen abstraction by the hydroxy-iron(III) species takes place to afford the oxindole.

The Guo and Duan ${ }^{11 a}$ and the Liang ${ }^{11 b}$ research groups have independently reported metal-free tert-butyl hydroperoxide mediated oxidative hydroxyalkylarylation of activated alkenes with $\mathrm{C}\left(\mathrm{sp}^{3}\right)-\mathrm{H}$ bonds adjacent to a hydroxy group (secondary or tertiary alcohols) for the assembly of hydroxy-containing oxindoles (Scheme 10). Mechanistic studies revealed that the $\alpha$-hydroxy carbon radical is initially formed from the $\alpha-\mathrm{C}\left(\mathrm{sp}^{3}\right)-\mathrm{H}$ bond cleavage, followed by intramolcular cyclization involving the aromatic $\mathrm{C}\left(\mathrm{sp}^{2}\right)-$ $\mathrm{H}$ radical coupling. Notably, the obtained hydroxy-containing oxindoles can be used for further transformations to give diverse indole-alkaloid structural motifs. 


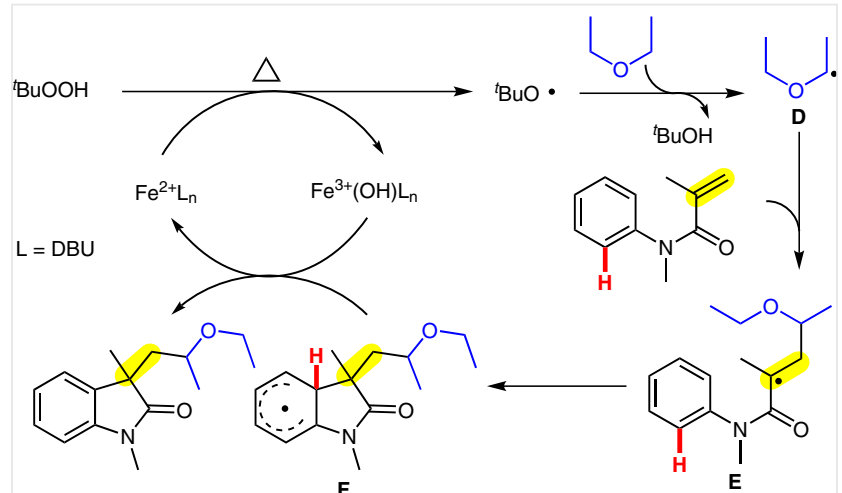

Scheme 9 Possible mechanism for the Fe-catalyzed 1,2-alkylarylation of alkenes with a $\mathrm{C}\left(\mathrm{sp}^{3}\right)-\mathrm{H}$ bond adjacent to a heteroatom

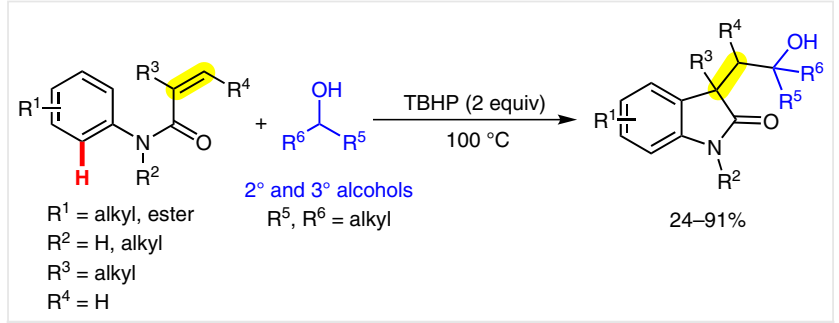

Scheme 10 1,2-Alkylarylation of alkenes with $\mathrm{C}\left(\mathrm{sp}^{3}\right)$ - $\mathrm{H}$ bonds adjacent to a hydroxy group

Generally, benzylic $\mathrm{C}\left(\mathrm{sp}^{3}\right)-\mathrm{H}$ bonds are highly reactive. Therefore, $\mathrm{Li}$ and co-workers attempted to extend the oxidative radical coupling protocol to simple benzylic $\mathrm{C}\left(\mathrm{sp}^{3}\right)-\mathrm{H}$ bonds. As expected, in the absence of metal catalysts, the reactions of activated alkenes with benzylic $\mathrm{C}\left(\mathrm{sp}^{3}\right)-\mathrm{H}$ bonds and di-tert-butyl peroxide (DTBP) were successful, albeit with lower yields (Scheme 11). ${ }^{12}$ It was found that Lewis acids could improve the reaction, and iridium(III) chloride was preferred. Meanwhile, the Guo and Duan research group reported a similar version of the 1,2-alkylarylation of alkenes with benzylic $\mathrm{C}\left(\mathrm{sp}^{3}\right)-\mathrm{H}$ bonds using a catalytic system comprised of copper(I) oxide and tert-butyl peroxybenzoate (TBPB). ${ }^{13}$

A novel copper-catalyzed oxidative 1,2-alkylarylation of alkenes with a range of simple alkanes, including cyclic alkanes, linear alkanes and arylmethanes, by using dicumyl peroxide (DCP) as oxidant has been reported by Liu and coworkers (Scheme 12). ${ }^{14}$ This method not only provided a useful method for the synthesis of alkyl-substituted oxindoles, but also presented a new strategy for selective functionalization of simple alkanes by way of a free-radical cascade process.
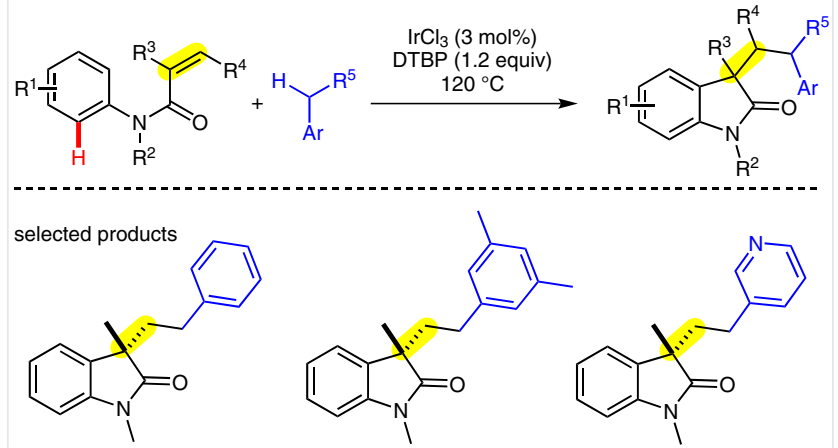

$83 \%$ (without $\mathrm{IrCl}_{3}: 53 \%$ )<smiles>Cc1cc(C)cc(CC[C@@]2(C)C(=O)N(C)c3ccccc32)c1</smiles><smiles>CN1C(=O)[C@](C)(CCc2cccnc2)c2ccccc21</smiles><smiles>Cn1c(=O)n(C)c2ccccc21</smiles><smiles></smiles><smiles>Cc1cc(C)cc(CCC2C(=O)N(C)c3ccccc32)c1</smiles>

Scheme 11 1,2-Alkylarylation of alkenes with benzylic $\mathrm{C}\left(\mathrm{sp}^{3}\right)$ - $\mathrm{H}$ bonds

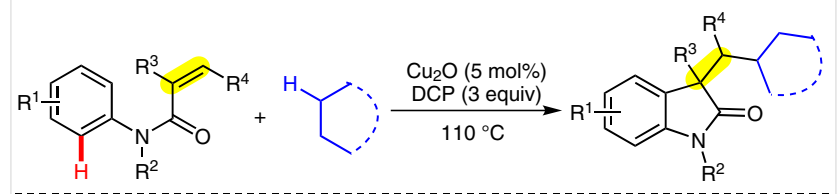

selected products<smiles>CN1C(=O)C(C)(CC2CCCCC2)c2ccccc21</smiles><smiles>CC1(CC2CCCCCC2)C(=O)N([13CH3])c2ccccc21</smiles><smiles>CCCCC(I)CC1(C)C(=O)N(C)c2ccc(Cl)cc21</smiles>

$66 \%(1 / 2 / 3=1: 6: 4)$<smiles>CC1(CCc2ccccc2)C(=O)N(C(C)(C)C)c2ccccc21</smiles>

Scheme 12 1,2-Alkylarylation of alkens with simple alkanes

Remarkably, Guo, Duan and Wang documented that alkyl radicals could be generated by $\mathrm{C}-\mathrm{H}$ oxidative cleavage with a new oxidative system, comprised of silver nitrate and potassium persulfate, that was thus not limited to peroxide oxidants (Scheme 13$).{ }^{15}$ In this reaction, the 1,2-alkylarylation of activated alkenes with the $\alpha-\mathrm{C}\left(\mathrm{sp}^{3}\right)-\mathrm{H}$ bonds of 1,3-dicarbonyl compounds was successful in furnishing oxindoles in good yields. Notably, the same reaction conditions were also extended to the reaction with simple ketones. 




selected products<smiles>CC(=O)C(CC1(C)C(=O)N(C)c2ccccc21)C(C)=O</smiles><smiles>CC(=O)C(CC1(C)C(=O)N(C)c2ccccc21)C(=O)c1ccccc1</smiles><smiles>CN1C(=O)C(C)(CC2=C(O)CC(C)(C)CC2=O)c2ccccc21</smiles>

$80 \%$
$94 \%(\mathrm{dr}=1.3: 1)$

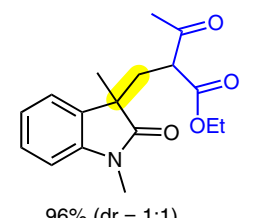

Scheme 13 Silver-catalyzed 1,2-alkylarylation of alkenes with $\alpha-C\left(s p^{3}\right)-H$ bonds in 1,3-dicarbonyl compounds

They proposed that the silver-catalyzed reaction proceeds through a sequential intermolecular radical addition and intramolecular radical substitution (Scheme 14). With the aid of silver nitrate and potassium persulfate, one of the $\alpha-\mathrm{C}\left(\mathrm{sp}^{3}\right)-\mathrm{H}$ bonds of the 1,3-dicarbonyl compound is split into alkyl radical intermediate $\mathbf{G}$ via a single-electrontransfer (SET) process, followed by addition across the alkene to produce intermediate $\mathbf{H}$. Cyclization of $\mathbf{H}$ occurs to afford intermediate I. Finally, hydrogen-abstraction of intermediate I with the silver catalyst gives the desired oxindole.



Scheme 14 Possible mechanism for the silver-catalyzed 1,2-alkylarylation of alkenes with 1,3-dicarbonyl compounds

They subsequently found that the alkyl radical intermediate $\mathbf{G}$ could be formed from the oxidation of 1,3-dicarbonyl compounds with potassium persulfate in the absence of silver salts, thus extending the method to build diversely functionalized spirooxindoles (Scheme 15$).{ }^{16}$ In the presence of potassium persulfate, hydroxymethylacrylamides successfully underwent the oxidative spirocyclization with 1,3-dicarbonyl compounds leading to the desired spirooxindoles. This process exhibited significant functional-group tolerance and high atom-economy.
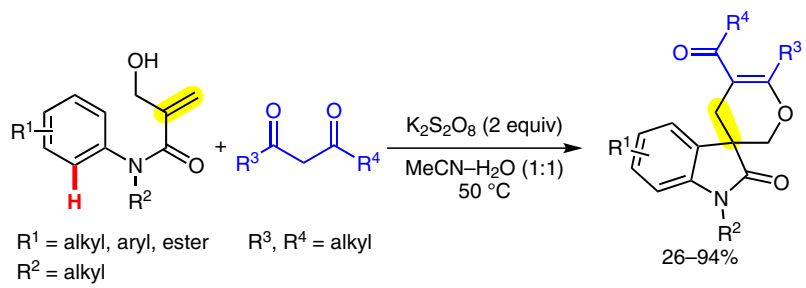

Scheme 15 Silver-free oxidative alkylspirocarbocyclization of hydroxymethylacrylamides with 1,3-dicarbonyl compounds

The 1,2-alkylarylations of alkenes with other carbon radicals that are not generated from the cleavage of the $\mathrm{C}-\mathrm{H}$ bonds have also been extensively explored. The single $\mathrm{C}\left(\mathrm{sp}^{2}\right)-\mathrm{H}$ oxidative radical coupling initiated by other carbon radicals are particularly fascinating because they open a new opportunity to incorporate various functional groups into the oxindole system through a radical strategy. In 2012, the Liu research group established a new radical strategy for the introduction of alkyl groups into the oxindole system by the decarboxylation of the (dialkylcarbonyloxyiodo)benzene $\left[\mathrm{PhI}(\mathrm{OCOR})_{2}\right] \quad$ (Scheme 16$) .{ }^{17}$ The results showed that the addition of an organocatalyst significantly improved the reaction yield. The reason may be that organocatalysts can stabilize radical intermediates $\mathbf{J}$ and $\mathbf{K}$, and make the deprotonation of intermediate $\mathbf{K}$ successful.

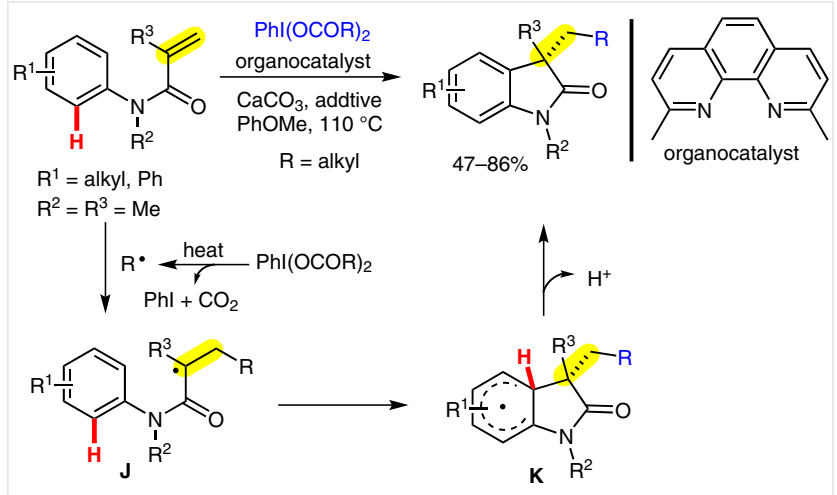

Scheme 16 1,2-Alkylarylation of alkenes with (dialkylcarbonyloxyiodo)benzenes 
Recently, the Zhu research group merged visible-light photoredox catalysis with $\mathrm{C}-\mathrm{H}$ oxidative radical coupling to achieve 1,2-alkylarylation with alkyl acids, in which oxidative decarboxylation of the alkyl acid by (diacetoxy)iodobenzene produced the alkyl radical (Scheme 17). ${ }^{18}$ The method was shown to be general and applicable to a range of readily available primary, secondary and tertiary aliphatic carboxylic acids. For example, bioactive lithocholic acid was a viable substrate for the tandem decarboxylation and 1,2-alkylarylation reaction.

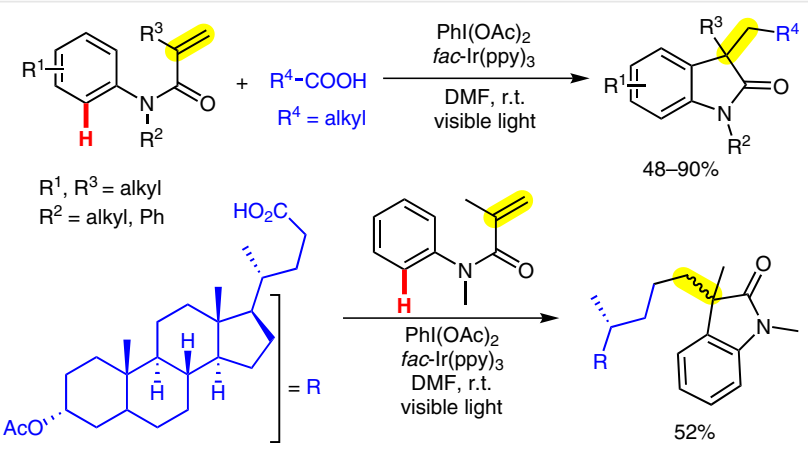

Scheme 17 1,2-Alkylarylation of alkenes with alkyl acids: the merging of visible-light photoredox catalysis with a $\mathrm{C}-\mathrm{H}$ oxidative radical coupling

Peroxides are usually used as the radical initiators or oxidants in organic synthesis, and are easily decomposed into different radical species under heating. However, methods to trap the radical species from peroxides are scare. The $\mathrm{Li}^{19 \mathrm{a}}$ and Cheng ${ }^{19 \mathrm{~b}}$ research groups independently reported that peroxides as alkyl sources underwent the 1,2-alkylarylation with activated alkenes (Scheme 18). The method allowed for the simultaneous formation of two carboncarbon bonds in the assembly of quaternary oxindoles.

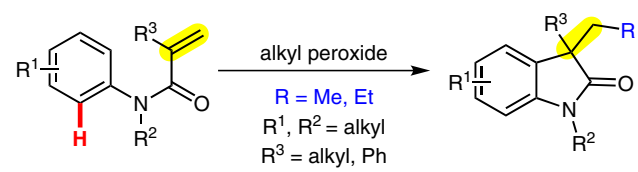

our conditions: $\mathrm{Fe}(\mathrm{OAc})_{2}$, DABCO, DMSO, $120^{\circ} \mathrm{C} \quad 46-66 \%$ Cheng conditions: $\mathrm{FeCl}_{2}$, benzene, $135^{\circ} \mathrm{C} \quad 49-84 \%$

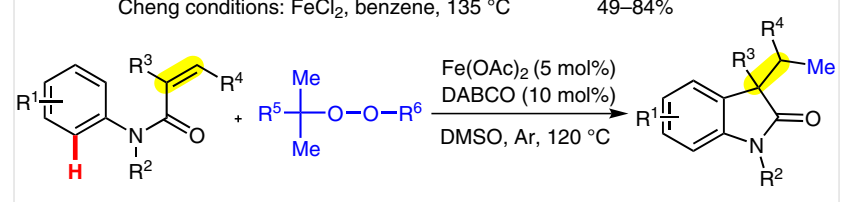



$62 \%$



$63 \%$

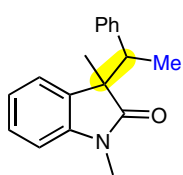

$66 \%$



$36 \%$
Scheme 18 1,2-Alkylarylation of alkenes with peroxides

\subsection{1,2-Aryltrifluoromethylation}

The trifluoromethyl group has been recognized as an important pharmacophore that can enhance the metabolic stability, lipophilicity, and bioavailability of the parent molecule. ${ }^{20}$ Considerable efforts have been paid to the development of methods for the incorporation of the trifluoromethyl group into bioactive molecules. As previously mentioned (Scheme 4), in 2012, Liu and co-workers reported a palladium-catalyzed oxidative aryltrifluoromethylation of activated alkenes for the construction of a variety of bioactive molecules containing trifluoromethylated oxindole moieties. The protocol included trimethyl(trifluorometh$\mathrm{yl}$ )silane with cesium fluoride as the source of the trifluoromethyl group, and (diacetoxy)iodobenzene as oxidant. ${ }^{8 b}$ Their preliminary mechanistic studies indicated that the reaction proceeds through initial arylpalladation of the alkene, followed by sequential oxidation and reductive elimination of $\mathrm{C}\left(\mathrm{sp}^{3}\right)$-trifluoromethylpalladium(IV) species to provide the oxindole products.

In recent years, much attention has been given to the use of the trifluoromethyl radical in the preparation of trifluoromethyl-containing compounds; however, successful examples are rare. In light of this, the 1,2-aryltrifluoromethylation of alkenes with the trifluoromethyl radical has been developed using different trifluoromethyl radical resources, such as sodium triflinate (the Langlois reagent) and trimethyl(trifluoromethyl)silane (Scheme 19). ${ }^{21}$ Interestingly, Tan and co-workers expanded the scope of reagents to include zinc difluoromethanesulfinate $\left(\mathrm{ZnSO}_{2} \mathrm{CF}_{2} \mathrm{H}\right)$ for the synthesis of difluoromethyl-containing oxindoles. ${ }^{22}$ The Wang research group also employed iododifluoromethyl phenyl sulfone $\left(\mathrm{PhSO}_{2} \mathrm{CF}_{2} \mathrm{I}\right)$ to generate the (phenylsulfonyl)difluoromethyl radical to access (phenylsulfonyl)difluoromethyl-containing oxindoles using the greener combination of ferrocene as catalyst and hydrogen peroxide as oxidant. ${ }^{23}$ Notably, these radical 1,2-aryltrifluoromethylation approaches not only exhibit high chemoselectivity for this transformation but also expand the substrate scope that is difficult to access by known transitionmetal-catalyzed methods, thus reflecting the synthetic utility of these transformations.



Scheme 19 1,2-Aryltrifluoromethylation of alkenes with the trifluoromethyl radical 
Following the increased interest in the trifluoromethyl radical strategy, Nevado and co-workers described a new copper-catalyzed sequential trifluoromethylation, 1,4-aryl migration, desulfonylation and $\mathrm{C}\left(\mathrm{sp}^{2}\right)-\mathrm{N}$ bond formation, involving conjugated tosyl amides and Togni's reagent as the reaction partners for the synthesis of trifluoromethylcontaining oxindoles (Scheme 20). ${ }^{24}$ The chemoselectivity depended on the N-substituent. The desired oxindoles were assembled when $\mathrm{N}$-alkyl tosyl amides were used. A crossover experiment was designed to determine whether the aryl migration is an intra- or intermolecular process, and the results showed that this method proceeds via intramolecular migration.

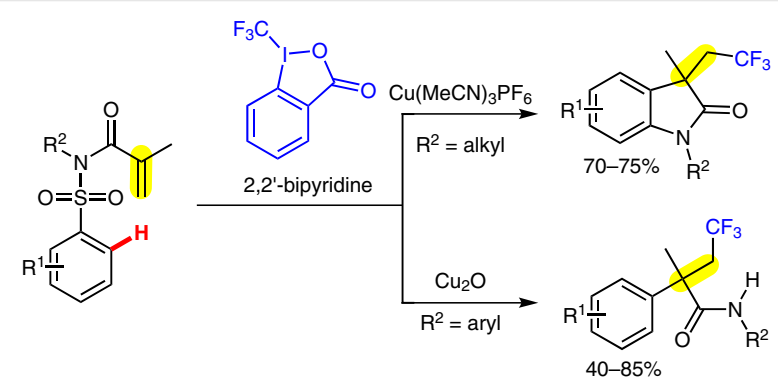

Scheme 20 Copper-catalyzed 1,2-aryltrifluoromethylation of alkenes through 1,4-aryl migration and desulfonylation

They proposed that the copper catalyst seemed to activate Togni's reagent, generating a highly reactive trifluoromethyl- and copper(II)-containing radical intermediate $\mathbf{L}$ which interacts with the activated alkene to give a new $\mathrm{C}\left(\mathrm{sp}^{3}\right)-\mathrm{CF}_{3}$ bond and $\alpha$-alkyl radical intermediate $\mathbf{M}$ (Scheme 21). A 5-ipso cyclization then takes place on the sulfonyl aromatic ring, generating aryl radical $\mathbf{N}$, followed by rapid desulfonylation to form the key amidyl radical in- termediate $\mathbf{0}$ with a new $\mathrm{C}\left(\mathrm{sp}^{2}\right)-\mathrm{C}\left(\mathrm{sp}^{3}\right)$ bond. Hydrogen abstraction of amidyl radical $\mathbf{O}$ occurs to give the trifluoromethylated amide, a process that seems to be favored for substrates with an aryl group (the substituent $\mathrm{R}^{2}$ ) on the nitrogen. In contrast, a more electron-donating alkyl moiety on the nitrogen triggers the oxidation of the radical to give copper enolate $\mathbf{P}$, and subsequent trapping by the aromatic ring leads to the oxindole.

\subsection{1,2-Carbonylarylation}

Carbonyl $\mathrm{C}\left(\mathrm{sp}^{2}\right)-\mathrm{H}$ bonds are highly reactive chemical bonds that are widely utilized in synthesis, ${ }^{25}$ and carbonylcontaining oxindoles are common structural motifs in pharmaceutical agents and natural products as well as being versatile intermediates in organic synthesis. Li and coworkers reported a metal-free 1,2-difunctionalization of alkenes with a range of carbonyl $\mathrm{C}\left(\mathrm{sp}^{2}\right)-\mathrm{H}$ bonds, namely aldehydes, formates and formamides (Scheme 22). ${ }^{26}$ In the presence of tert-butyl hydroperoxide, the oxidative tandem couplings of activated alkenes with carbonyl $\mathrm{C}\left(\mathrm{sp}^{2}\right)-\mathrm{H}$ bonds and aryl $\mathrm{C}\left(\mathrm{sp}^{2}\right)-\mathrm{H}$ bonds were carried out smoothly, offering 3-(2-oxoethyl)indolin-2-ones in good yields.

To understand the mechanism, inter- and intramolecular kinetic isotope experiments were performed (Scheme 23): the arylation step may be compatible with either the $\mathrm{S}_{\mathrm{E}} \mathrm{Ar}$ mechanism or the free-radical mechanism because no kinetic isotope effect was observed $\left(k_{H} / k_{D}=1.0\right)$. The freeradical mechanism was supported by the control experiments carried out with radical inhibitors: a stoichiometric amount of radical inhibitor, such as TEMPO or 2,6-di-tertbutylphenol, was added to the difunctionalization reaction mixture, and no reaction took place. A large kinetic isotope effect $\left(k_{H} / k_{D}=5.0\right)$ was observed in the reaction of amide

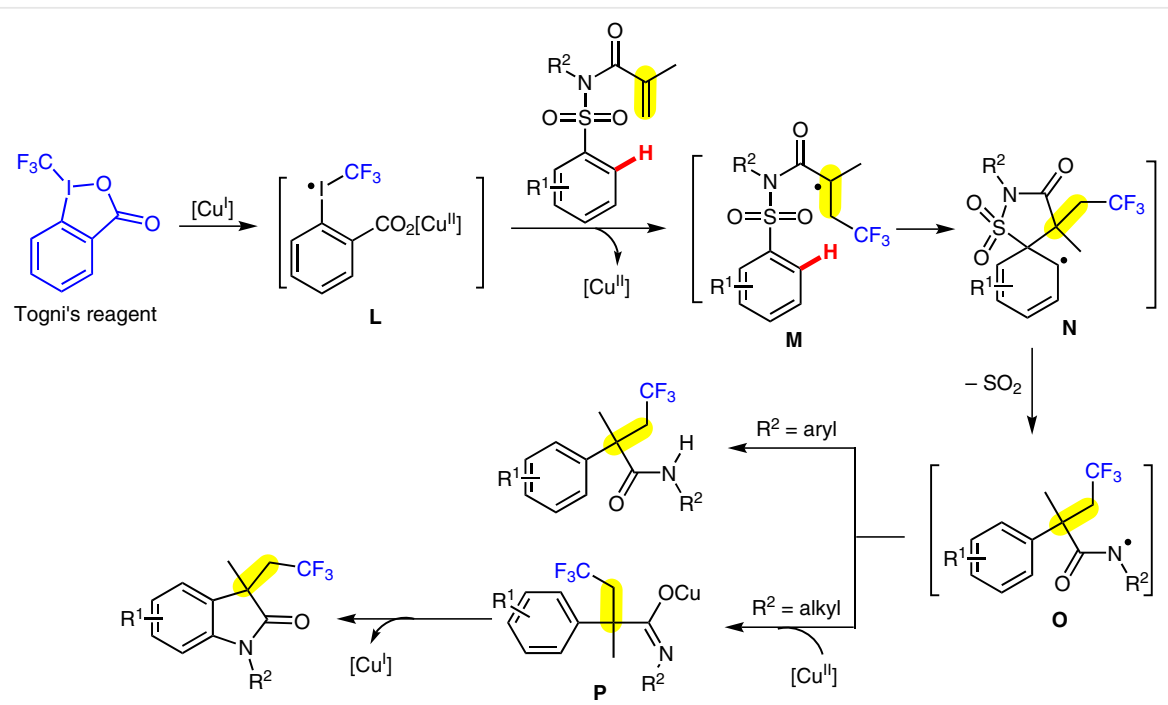

Scheme 21 Possible mechanism for the 1,2-aryltrifluoromethylation of alkenes through 1,4-aryl migration and desulfonylation 


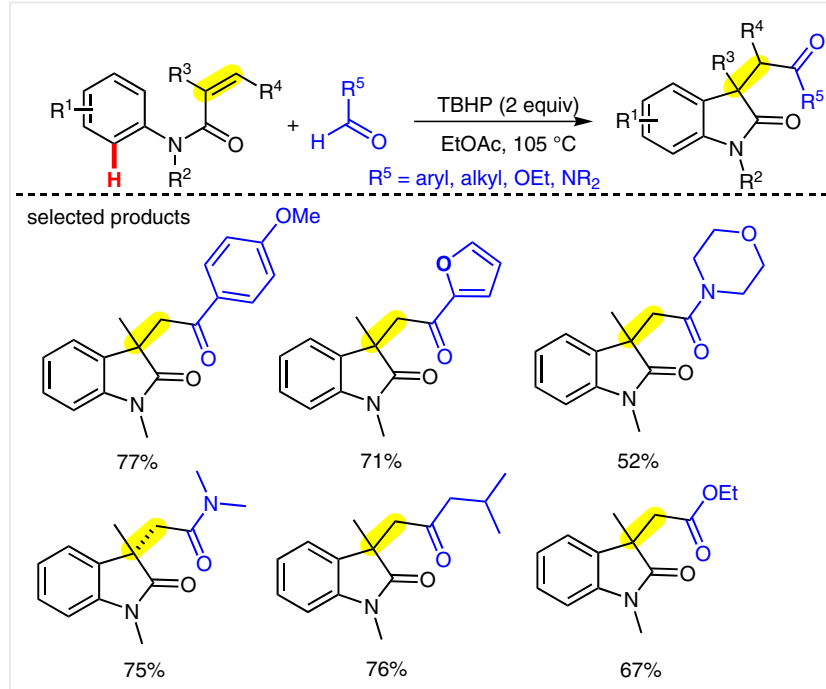

Scheme 22 1,2-Carbonylarylation of alkenes with carbonyl C(sp $\left.{ }^{2}\right)-\mathrm{H}$ bonds

with $\mathrm{N}, \mathrm{N}$-dimethylformamide and $\mathrm{N}, \mathrm{N}$-dimethylformamide- $d_{7}$, suggesting that the cleavage of the carbonyl carbon-hydrogen bond is the rate-limiting step.

Accordingly, the radical mechanism outlined in Scheme 23 was proposed for this 1,2-carbonylarylation. The aldehydic hydrogen is easily abstracted by an alkyloxy or hydroxy radical, generated in situ from tert-butyl hydroperoxide under heating, to yield aldehydic radical $\mathbf{Q}$. Addition across the carbon-carbon double bond affords radical intermediate $\mathbf{R}$, then intramolecular cyclization with an aryl ring takes place to give radical intermediate $\mathbf{S}$. Finally, abstraction of an aryl hydrogen by tert-butyl hydroperoxide delivers the 3-(2-oxoethyl)indolin-2-one.

Prompted by these results, and because primary alcohols can be easily oxidized into the corresponding aldehydes, Li and co-workers turned their attention to the oxidative tandem coupling of activated alkenes with primary alcohols. As expected, 1,2-carbonylarylation of $\mathrm{N}$-arylacrylamides with alcohols was achieved in the presence of iron(II) acetate and tert-butyl hydroperoxide (Scheme 24). ${ }^{27}$ Notably, the desired oxindole was constructed in $28 \%$ yield even without the iron catalyst.

Guo, Duan and Wang showed that carbonyl radicals were produced from the oxidative decarboxylation of $\alpha$ oxocarboxylic acids using the catalytic system comprised of silver and potassium persulfate (Scheme 25 ). ${ }^{28}$ In the presence of silver nitrate and potassium persulfate, a wide range of carbonyl-containing oxindoles were constructed in good to excellent yields through the radical decarboxylative 1,2-carbonylarylation of alkenes with easily available $\alpha$ oxocarboxylic acids. This transformation proceeded well under mild reaction conditions and exhibited excellent functional-group tolerance.

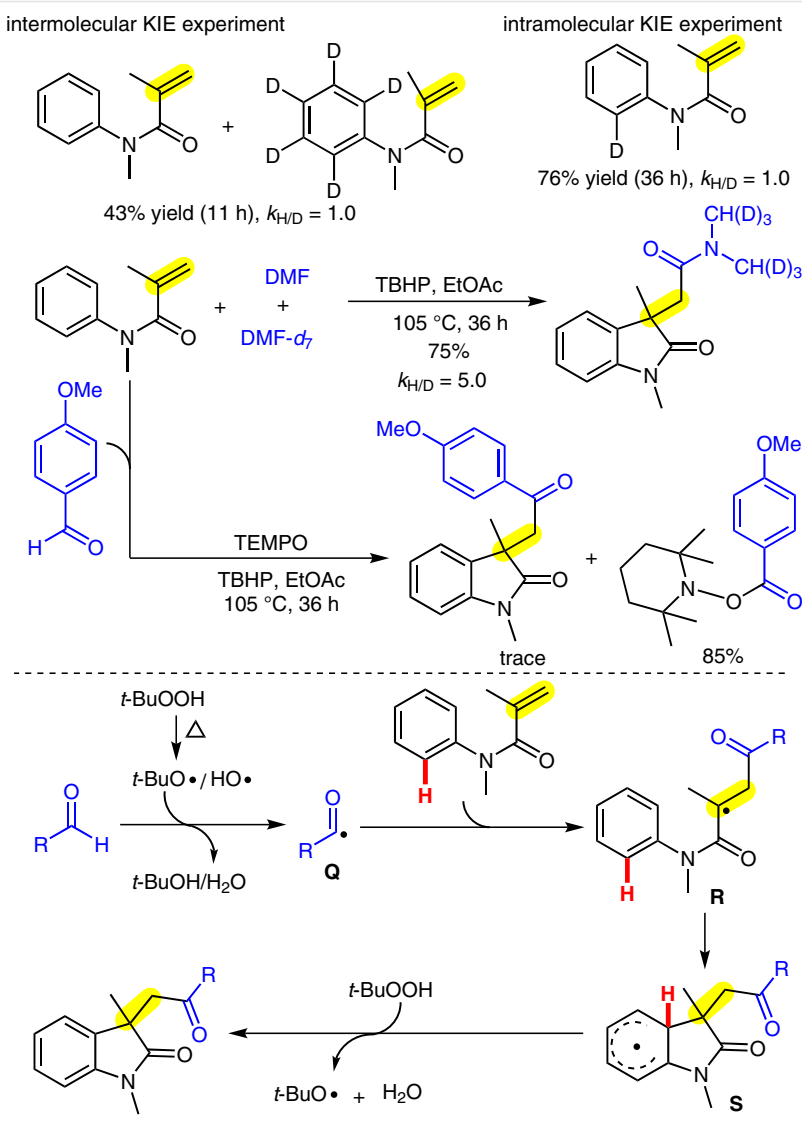

Scheme 23 Control experiments and possible mechanism for the 1,2carbonylarylation of alkenes with carbonyl $\mathrm{C}\left(\mathrm{sp}^{2}\right)-\mathrm{H}$ bonds



77\% (without Fe: $28 \%$ )

Scheme 24 Iron-catalyzed 1,2-carbonylarylation of alkenes with primary alcohols

$$
\begin{aligned}
& R^{1}=\text { alkyl, aryl, ester } \\
& R^{2}=\text { alkyl } \\
& R^{3}=\text { alkyl, } \mathrm{Ph}
\end{aligned}
$$

Scheme 25 Silver-catalyzed 1,2-acylarylation of alkenes with $\alpha$-oxocarboxylic acids

A possible mechanism was postulated for this tandem reaction (Scheme 26). Initially, the $\alpha$-oxocarboxylic acid is converted into the corresponding aldehydic radical $\mathbf{Q}$ and the remaining steps are similar to those in the 1,2-carbonylarylation between alkenes and carbonyl $\mathrm{C}\left(\mathrm{sp}^{2}\right)-\mathrm{H}$ bonds. ${ }^{23}$ 


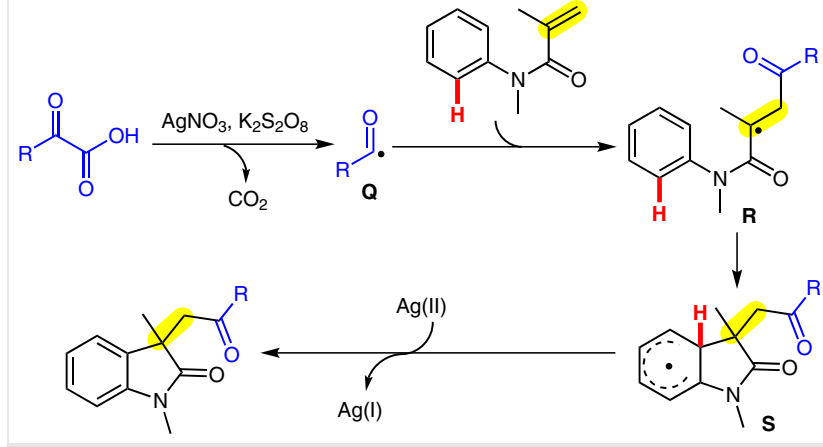

Scheme 26 Possible mechanism for 1,2-acylarylation of alkenes with $\alpha$-oxocarboxylic acids

In recent studies by $\mathrm{Li}, \mathrm{Du}$ and co-workers ${ }^{29 \mathrm{a}}$ and by Chen, $\mathrm{Yu}$ and co-workers, ${ }^{29 \mathrm{~b}}$ the alkoxycarbonyl radicals were formed readily by the oxidative cleavage of the carbon-nitrogen bond of a carbazate (Scheme 27). They used carbazates as easily accessible and safe alkoxycarbonyl radical precursors for the 1,2-alkoxycarbonylarylation of $\mathrm{N}$-aryl acrylamides. The method provides a general and practical method for the construction of alkoxycarbonylated oxindoles. Moreover, the use of economical and environmentally benign iron as the catalyst makes this transformation more sustainable and practical.


Chen/Yu conditions: $\mathrm{FeCl}_{2} \cdot 4 \mathrm{H}_{2} \mathrm{O}$, 4-cyanopyridine, TBHP, EtOAc, $80{ }^{\circ} \mathrm{C} \quad 40-92 \%$

Scheme 27 Iron-catalyzed 1,2-alkoxycarbonylarylation of $\mathrm{N}$-aryl acrylamides with carbazates

\section{Synthesis of Oxindoles via Carbohetero- functionalization of Alkenes}

\subsection{1,2-Azidoarylation or 1,2-Arylnitration}

The $\mathrm{C}\left(\mathrm{sp}^{2}\right)-\mathrm{H}$ oxidative radical coupling strategy is applicable to the carbo-heterofunctionalization of alkenes with two type of nitrogen radicals: azidyl and nitro radical. In 2013, Antonchick and co-workers reported an unprecedented metal-free azidoarylation of alkenes with azidyl radicals that were generated by the oxidation of azidotrimethylsilane in the presence of hypervalent iodine(III) reagents at ambient temperature (Scheme 28). ${ }^{30}$ In the presence of phenyliodine bis(trifluoroacetate) and azidotrimethylsilane, a variety of $\mathrm{N}$-arylacrylamides delivered the corresponding azide-containing oxindoles in good yields. It was noted that 2-oxindoles with an appended azide group could be used to create further molecular complexity around the privileged scaffold of 2-oxindoles, or could be applied for bioorthogonal transformation under physiological conditions.

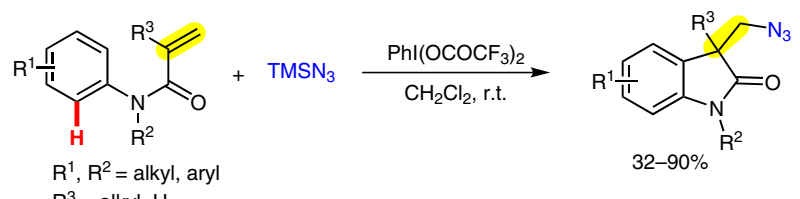

Scheme 28 Metal-free azidoarylation of alkenes with azidotrimethylsilane

The reaction is thought to proceed through a double ligand exchange between phenyliodine bis(trifluoroacetate) and azidotrimethylsilane to afford intermediate $\mathbf{T}$ (Scheme 29). The latter is not stable and easily undergoes thermal homolytic cleavage to form an azide radical, which then attacks the alkene to give radical $\mathbf{U}$. Intramolecular trapping with the arene gives rise to intermediate $\mathbf{V}$, which then undergoes rearomatization to produce the oxindole.

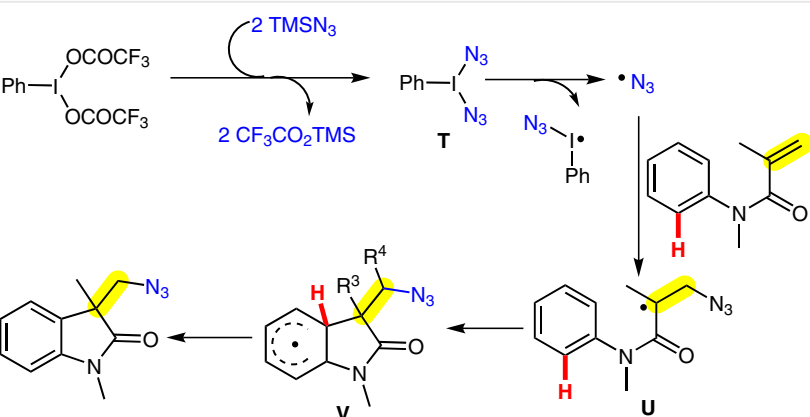

Scheme 29 Possible mechanism for the azidoarylation of alkenes with azidotrimethylsilane

Around that same time, the Yang ${ }^{31 a}$ and Jiao $^{31 b}$ research groups independently documented a new strategy for the formation of the azidyl radical through the oxidation of azidotrimethylsilane with silver nitrate and oxidizing reagents such as zirconium nitrate or cerium(IV) sulfate (Scheme 30). Through their methods, various azide-containing oxindoles were prepared smoothly in moderate to good yields.



Scheme 30 Possible mechanism for the silver-catalyzed azidoarylation of alkenes with azidotrimethylsilane 
Very recently, Zhang and Qiu reported a metal-free carboazidation of acrylamides with sodium azide in the presence of potassium persulfate as oxidant. This transformation exhibited excellent functional-group tolerance. ${ }^{32}$

Nitro compounds are important structural features of pharmaceuticals and functional materials, as well as being versatile intermediates in synthesis. ${ }^{33} \mathrm{~A}$ nitro radical for the 1,2-carbonitration of alkenes was first introduced by Yang and co-workers in 2013 (Scheme 31). ${ }^{34}$ This protocol provided a new shortcut for the assembly of nitro-containing oxindoles, in which the nitro radical is formed from the oxidation of sodium nitrite by potassium persulfate.

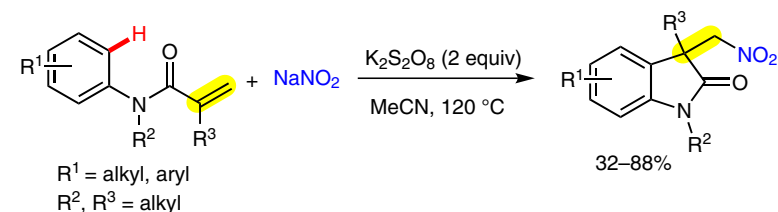

Scheme 31 1,2-Arylnitration of alkenes with sodium nitrite

Jiao and co-workers tested the use of tert-butyl nitrite as the nitrogen dioxide source for the carbonitration of alkenes, and were successful in accessing nitro-containing oxindoles under metal-free conditions (Scheme 32). ${ }^{35}$ Mechanistic studies indicated that both the nitric oxide radical $(\cdot \mathrm{NO})$ and the nitrogen dioxide radical $\left(\cdot \mathrm{NO}_{2}\right)$ addition across the carbon-carbon double bond were involved in this transformation, and that nitrogen dioxide radical addition occurred prior to nitric oxide radical addition. Notably, this reaction was applicable to unactivated alkenes.

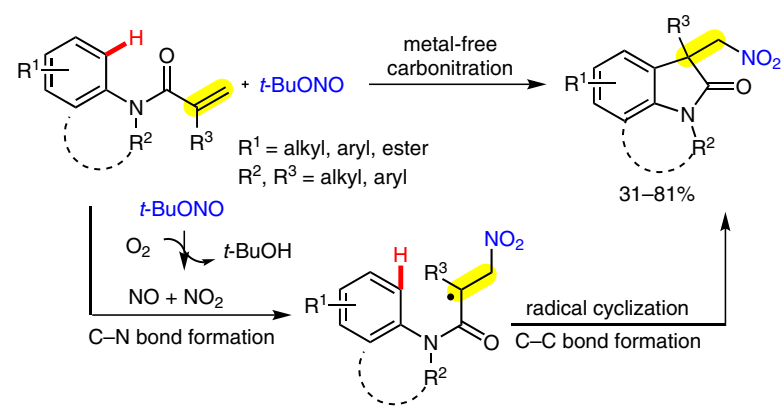

Scheme 32 Metal-free 1,2-arylnitration of alkenes with tert-butyl nitrite

\subsection{1,2-Arylsulfonylation}

The important roles of sulfur-containing compounds in pharmaceuticals and agrochemicals have inspired chemists to continue developing mild and efficient carbon-sulfur bond-forming methods. ${ }^{36} \mathrm{~A}$ pioneering work on the difunctionalization of alkenes with sulfonyl radicals was published in 2013 by Li, Xu and co-workers (Scheme 33). ${ }^{37}$ The oxidative 1,2-arylsulfonylation of activated alkenes with $p$ - toluenesulfonyl hydrazine proceeded using potassium iodide as catalyst, THBP as oxidant and 18-crown-6 as promoter, thus affording sulfonated oxindoles in satisfactory yields. This method uses the readily available $p$-toluenesulfonyl hydrazine as sulfonyl source by way of a radical strategy, and represents an environmentally benign access to oxindoles by using nontoxic potassium iodide as catalyst and water as medium.

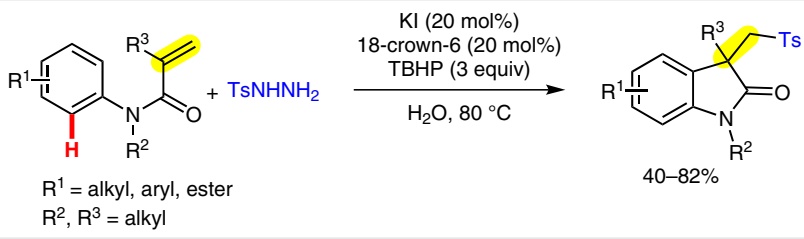

Scheme 33 1,2-Arylsulfonylation of activated alkenes with $p$-toluenesulfonyl hydrazine

Both potassium iodide and 18-crown-6 were proposed to accelerate the decomposition of tert-butyl hydroperoxide into the tert-butoxy radical (Scheme 34). The resultant abstraction of hydrogen from $p$-toluenesulfonyl hydrazine by the tert-butoxy radical generates a sulfonyl radical via the release of molecular nitrogen from intermediate radical $\mathbf{W}$. The addition of the sulfonyl radical to the alkene offers intermediate $\mathbf{X}$, and subsequent intamolecular cyclization with the arene produces intermediate $\mathbf{Y}$. Hydrogen abstraction of intermediate $\mathbf{Y}$ by tert-butyl hydroperoxide affords the sulfonated oxindole.

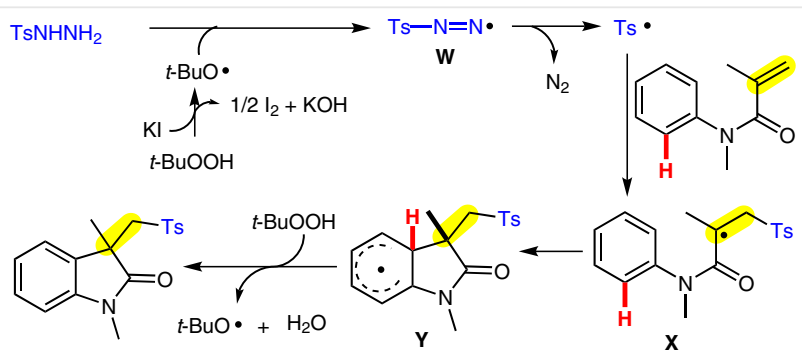

Scheme 34 Possible mechanism for the 1,2-arylsulfonylation of activated alkenes with $p$-toluenesulfonyl hydrazine

A similar conversion was subsequently illustrated by Kuang and co-workers, starting from $\mathrm{N}$-arylsulfonylacrylamides (Scheme 35). ${ }^{38}$ In the presence of copper(II) triflate and potassium persulfate, $p$-toluenesulfonyl hydrazine was converted into the arenesulfonyl radical, thus achieving the 1,2-arylsulfonylation of $\mathrm{N}$-arylsulfonylacrylamides through a sulfonylation, 5-ipso-cyclization, aryl migration, desulfonylation and amidyl radical cyclization cascade.

A example using sulfinic acids to form the sulfonyl radicals was developed by the Wang research group (Scheme 36). ${ }^{39}$ An important feature of this strategy is that only potassium persulfate was employed to oxidize the sulfinic 


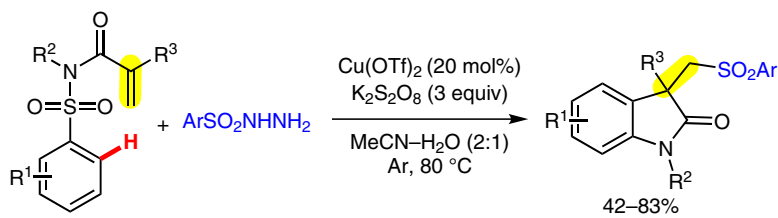

$$
\begin{aligned}
& \mathrm{R}^{1}=\text { alkyl, aryl } \\
& R^{2}, R^{3}=\text { alkyl }
\end{aligned}
$$

Scheme 35 1,2-Arylsulfonylation of activated alkenes with p-toluenesulfonyl hydrazine through aryl migration and desulfonylation

acid to produce the sulfonyl radical. Direct 1,2-arylsulfonylation of the $N$-arylacrylamide then led to the sulfonated oxindole.

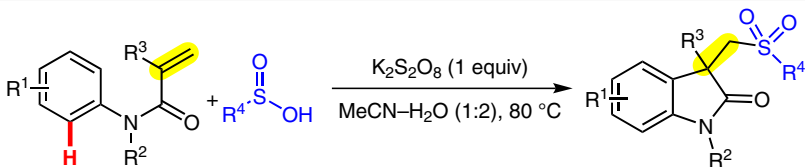

$$
\begin{aligned}
& R^{1}, R^{3}=\text { alkyl } \quad R^{4}=\text { aryl } \quad 54-93 \% \\
& \mathrm{R}^{2}=\mathrm{Me}, \mathrm{Ph}
\end{aligned}
$$

Scheme 36 1,2-Arylsulfonylation of activated alkenes with sulfinic acids

Very recently, Wang and co-workers reported a silvermediated 1,2-aryltrifluoromethylthiolation of activated alkenes with [(trifluoromethyl)thio]silver in the presence of potassium persulfate as oxidant (Scheme 37). ${ }^{40}$ This method allowed for the oxidative conversion of [(trifluoromethyl)thio]silver into (trifluoromethyl)thio radical by the oxidant, and provided a facile route to trifluoromethylthiolcontaining oxindoles.

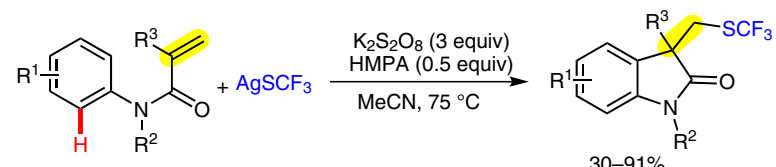

$$
\begin{aligned}
& \mathrm{R}^{1}=\text { alkyl, aryl, ester } \\
& \mathrm{R}^{2}, \mathrm{R}^{3}=\text { alkyl }
\end{aligned}
$$

Scheme 37 Silver-catalyzed 1,2-aryltrifluoromethylthiolation of alkenes with [(trifluoromethyl)thio]silver
A possible mechanism involving a radical process was proposed for this reaction (Scheme 38). Initially, oxidation of [(trifluoromethyl)thio]silver by potassium persulfate gives [(trifluoromethyl)thio]silver(II), which then forms the key (trifluoromethyl)thio radical $\mathbf{Z}$. The latter undergoes a sequential radical addition and cyclization with $\mathrm{N}$-arylacrylamide to form the final oxindole by way of radical intermediates $\mathbf{A A}$ and $\mathbf{A B}$.

\subsection{1,2-Arylphosphorylation}

Organophosphorus compounds are a prominent molecular class in organic chemistry and are widely distributed in pharmaceuticals and agrochemicals; considerable effort has been devoted to the development of new convenient and flexible methods for the formation of carbon-phosphorus bonds. ${ }^{41}$ The first application of the phosphine oxide radicals for the carbophosphorylation of alkenes was reported in 2013 by Yang and co-workers (Scheme 39). ${ }^{42} \mathrm{~A}$ number of phosphorylated oxindoles were synthesized by using a catalytic system comprised of silver nitrate and magnesium nitrate hexahydrate to trigger the radical carbophosphorylation of $\mathrm{N}$-arylacrylamides with phosphine oxides.



Scheme 39 Silver-catalyzed 1,2-arylphosphorylation of alkenes with phosphine oxides

Mechanistically, the $\mathrm{Ph}_{2} \mathrm{P}(\mathrm{O}) \mathrm{Ag}$ intermediate $\mathbf{A D}$, which is formed from the reaction of silver(I) with diphenylphosphine oxide, is proposed to play a crucial role in the alkene 1,2-arylphosphorylation process (Scheme 40 ). It may take place through two paths: one proceeds by the formation of diphenylphosphinoyl radical from $\mathbf{A D}$ and its addition across the alkene to produce $\mathbf{A F}$ (path $\mathrm{A}$ ), the other involves

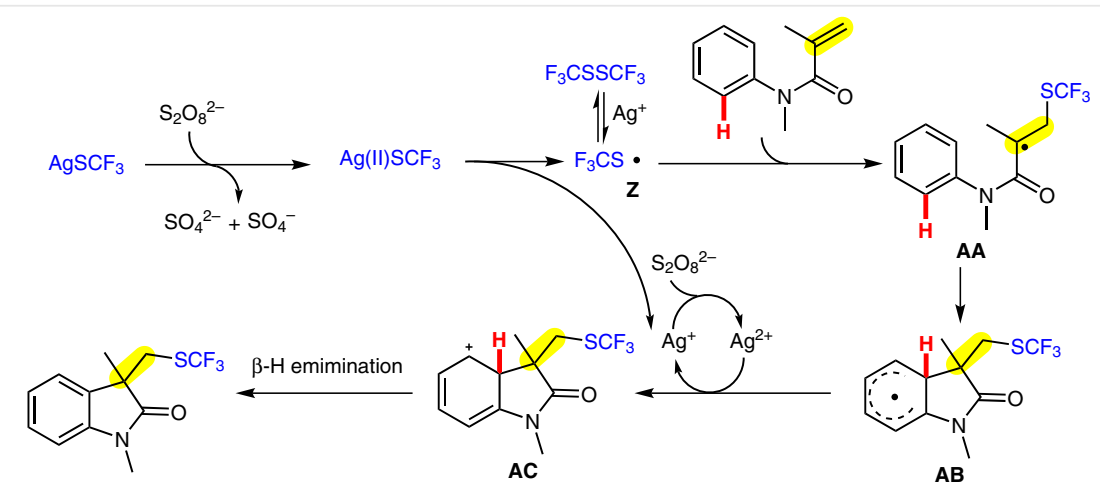

Scheme 38 Possible mechanism for the silver-catalyzed 1,2-aryltrifluoromethylthiolation of alkenes 
the addition of $\mathbf{A D}$ itself to the carbon-carbon double bond, leading to intermediate $\mathbf{A E}$ which is then oxidized to $\mathbf{A F}$. Both paths proceed from intermediate $\mathbf{A F}$.

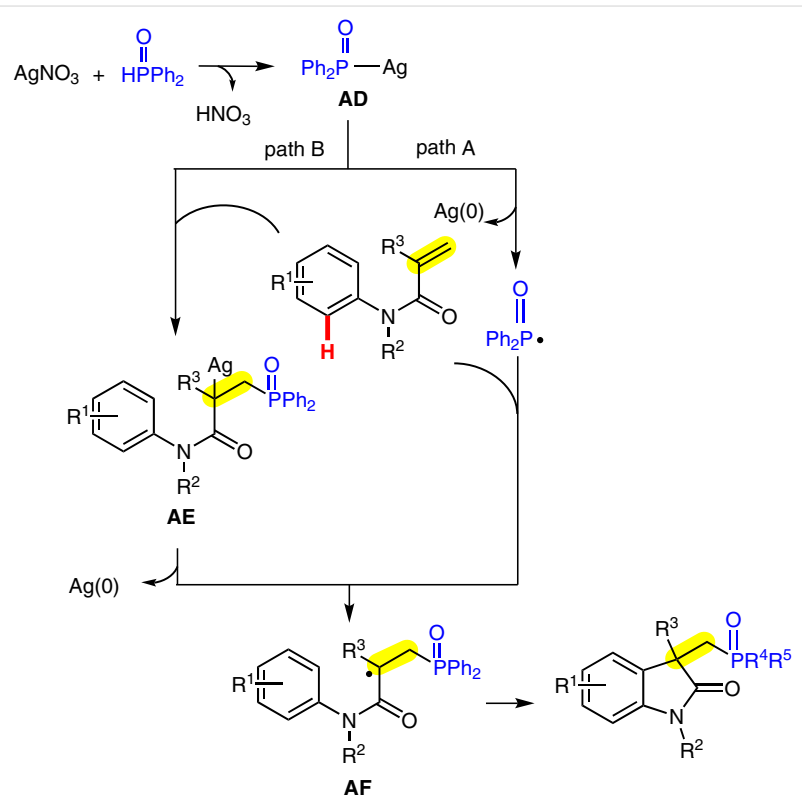

Scheme 40 Possible mechanism for the silver-catalyzed 1,2-arylphosphorylation of alkenes with phosphine oxides

The $\mathrm{Ph}_{2} \mathrm{P}(\mathrm{O})$ radical could also be formed from the oxidation of phosphine oxides by potassium persulfate in the absence of a silver catalyst (Scheme 41 ). ${ }^{43}$ This arylphosphination of $\mathrm{N}$-arylacrylamides with phosphine oxides was used to assemble various phosphorus-containing oxindoles in high yields.

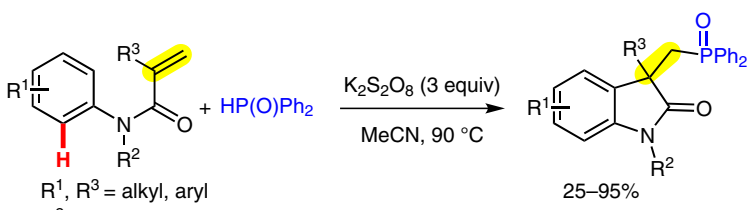

$$
\begin{aligned}
& \mathrm{R}^{2}=\text { alkyl }
\end{aligned}
$$

Scheme 41 Transition-metal-free 1,2-arylphosphorylation of alkenes with phosphine oxides

\section{Conclusion}

In the last five years, activated alkene 1,2-difunctionalization reactions involving a $\mathrm{C}-\mathrm{H}$ oxidative coupling process have been widely used in the synthesis of heterocyclic compounds, in particular oxindoles and their derivatives. This review summarized recent efforts along these lines. These new methods are triggered by carbon or heteroatom radicals, thus easily incorporating many functional groups, including alkyl, hydroxyl, carbonyl, trifluoromethyl, azidyl, nitro, sulfonyl, (trifluoromethyl)thio and phosphoryl groups, into the oxindole framework. Owing to their high efficiency, high step- and atom-economy, excellent functional-group tolerance and broad substrate scope, the current $\mathrm{C}-\mathrm{H}$ oxidative radical coupling reactions will have widespread applications in the pharmaceutical, agrochemical and materials fields.

Despite significant progress in the use of $\mathrm{C}-\mathrm{H}$ oxidative radical coupling for the synthesis of oxindoles, oxidant dependence is very prominent in the alkene difunctionalization transformations, and stoichiometric amounts of nongreen oxidants, such as peroxides, potassium persulfate, hypervalent iodines or tert-butyl nitrite, are necessary in all cases. Moreover, the substrate scope in many cases is limited to activated alkenes, and the related mechanisms need to be explored in more detail. Therefore, the development of new, efficient $\mathrm{C}-\mathrm{H}$ oxidative radical coupling strategies involving the use of green oxidants (e.g., $\mathrm{O}_{2}$, hydrogen peroxide or other sustainable oxidants) is highly desirable. The important opportunities and challenges in this field will involve determining how to achieve selective coupling of unactivated alkyl or aryl $\mathrm{C}-\mathrm{H}$ bonds. In addition, more new functional radicals also need to be designed and discovered, in order to initiate research into further unexplored $\mathrm{C}-\mathrm{H}$ oxidative radical coupling methods.

\section{Acknowledgment}

This research was supported by the NSFC (Nos. 21402046, 21172060 and 21472039) and Hunan Provincial Natural Science Foundation of China (No. 13JJ2018). Dr. R.-J. S. also thanks the Fundamental Research Funds for the Central Universities.

\section{References}

(1) For selected reviews and papers, see: (a) Lin, H.; Danishefsky, S. J. Angew. Chem. Int. Ed. 2003, 42, 36. (b) Emura, T.; Esaki, T.; Tachibana, K.; Shimizu, M. J. Org. Chem. 2006, 71, 8559. (c) Dalpozzo, R.; Bartoli, G.; Bencivenni, G. Chem. Soc. Rev. 2012, 41, 7247. (d) Singh, G. S.; Desta, Z. Y. Chem. Rev. 2012, 112, 6104. (e) Shen, K.; Liu, X.; Lin, L.; Feng, X. Chem. Sci. 2012, 3, 327. (f) Ohmatsu, K.; Ando, Y.; Ooi, T. J. Am. Chem. Soc. 2013, 135, 18706.

(2) For selected examples, see: (a) Hennessy, E. J.; Buchwald, S. L. J. Am. Chem. Soc. 2003, 125, 12084. (b) Galliford, C. V.; Scheidt, K. A. Angew. Chem. Int. Ed. 2007, 46, 8748. (c) Jia, Y.-X.; Kündig, E. P. Angew. Chem. Int. Ed. 2009, 48, 1636. (d) Piou, T.; Neuville, L.; Zhu, J. Angew. Chem. Int. Ed. 2012, 51, 11561.

(3) For selected reviews on difunctionalization of alkenes, see: (a) Kolb, H. C.; van Nieuwenhze, M. S.; Sharpless, K. B. Chem. Rev. 1994, 94, 2483. (b) Beccalli, E. M.; Broggini, G.; Martinelli, M.; Sottocornola, S. Chem. Rev. 2007, 107, 5318. (c) Jacques, B.; Muniz, K. In Catalyzed Carbon-Heteroatom Bond Formation; Yudin, A. K., Ed.; Wiley-VCH: Weinheim, 2010, 119. (d) McDonald, R. I.; Liu, G.; Stahl, S. S. Chem. Rev. 2011, 111, 2981. (e) Chen, J.-R.; Yu, X.-Y.; Xiao, W.-J. Synthesis 2015, 47, 604.

(4) Evans, P.; Grigg, R.; Ramzan, M. I.; Sridharan, V.; York, M. Tetrahedron Lett. 1999, 40, 3021. 
(5) Anwar, U.; Casaschi, A.; Grigg, R.; Sansano, J. M. Tetrahedron 2001, 57, 1361.

(6) Pinto, A.; Jia, Y.; Neuville, L.; Zhu, J. Chem. Eur. J. 2007, 13, 961.

(7) Jaegli, S. P.; Dufour, J.; Wei, H.-L.; Piou, T.; Duan, X.-H.; Vors, J.P.; Neuville, L.; Zhu, J. Org. Lett. 2010, 12, 4498.

(8) (a) Wu, T.; Mu, X.; Liu, G. Angew. Chem. Int. Ed. 2011, 50, 12578. (b) Mu, X.; Wu, T.; Wang, H.-Y.; Guo, Y.-L.; Liu, G. J. Am. Chem. Soc. 2012, 134, 878.

(9) (a) Murakami, M.; Ito, Y. In Topics in Organometallic Chemistry; Vol. 3; Murai, S., Ed.; Springer-Verlag: New York, 1999, 97-129. (b) Rybtchinski, B.; Milstein, D. Angew. Chem. Int. Ed. 1999, 38, 871. (c) Sundermann, A.; Uzan, O.; Milstein, D.; Martin, J. M. L. J. Am. Chem. Soc. 2000, 122, 7095. (d) Gao, X.; Woo, S. K.; Krische, M. J. J. Am. Chem. Soc. 2013, 135, 4223. (e) Del Valle, D. J.; Krische, M. J. J. Am. Chem. Soc. 2013, 135, 10986. (f) Barroso, R.; Valencia, R. A.; Cabal, M.-P.; Valdés, C. Org. Lett. 2014, 16, 2264.

(10) Wei, W.-T.; Zhou, M.-B.; Fan, J.-H.; Liu, W.; Song, R.-J.; Liu, Y.; Hu, M.; Xie, P.; Li, J.-H. Angew. Chem. Int. Ed. 2013, 52, 3638.

(11) (a) Meng, Y.; Guo, L.-N.; Wang, H.; Duan, X.-H. Chem. Commun. 2013, 49, 7540. (b) Zhou, Z.-Z.; Hua, H.-L.; Luo, J.-Y.; Chen, Z.-S.; Zhou, P.-X.; Liu, X.-Y.; Liang, Y.-M. Tetrahedron 2013, 69, 10030.

(12) Zhou, M.-B.; Wang, C.-Y.; Song, R.-J.; Liu, Y.; Wei, W.-T.; Li, J.-H. Chem. Commun. 2013, 49, 10817.

(13) Zhou, S.-L.; Guo, L.-N.; Wang, H.; Duan, X.-H. Chem. Eur. J. 2013, $19,12970$.

(14) Li, Z.-J.; Zhang, Y.; Zhang, L.-Z.; Liu, Z.-Q. Org. Lett. 2014, 16, 382.

(15) Wang, H.; Guo, L.-N.; Duan, X.-H. Chem. Commun. 2013, 49, 10370.

(16) Wang, H.; Guo, L.-N.; Duan, X.-H. Org. Lett. 2013, 15, 5254.

(17) Wu, T.; Zhang, H.; Liu, G. Tetrahedron 2012, 68, 5229.

(18) Xie, J.; Xu, P.; Li, H.; Xue, Q.; Jin, H.; Cheng, Y.; Zhu, C. Chem. Commun. 2013, 49, 5672.

(19) (a) Fan, J.-H.; Zhou, M.-B.; Liu, Y.; Wei, W.-T.; Ouyang, X.-H.; Song, R.-J.; Li, J.-H. Synlett 2014, 25, 657. (b) Dai, Q.; Yu, J.; Jiang, Y.; Guo, S.; Yang, H.; Cheng, J. Chem. Commun. 2014, 50, 3865.

(20) (a) Smart, B. E. Chem. Rev. 1996, 96, 1555. (b) Shimizu, M.; Hiyama, T. Angew. Chem. Int. Ed. 2005, 44, 214. (c) Muller, C. K.; Faeh, C.; Diederich, F. Science 2007, 317, 1881. (d) Purser, S.; Moore, P. R.; Swallow, S.; Gouverneur, V. Chem. Soc. Rev. 2008, 37, 320. (e) Wang, F.; Wang, D.-H.; Mu, X.; Chen, P.-H.; Liu, G.-S. J. Am. Chem. Soc. 2014, 136, 10202.

(21) (a) Egami, H.; Shimizu, R.; Sodeoka, M. J. Fluorine Chem. 2013, 152, 51. (b) Li, L.; Deng, M.; Zheng, S.-C.; Xiong, Y.-P.; Tan, B.; Liu, X.-Y. Org. Lett. 2014, 16, 504. (c) Yang, F.; Klumphu, P.; Liang, Y.-M.; Lipshutz, B. H. Chem. Commun. 2014, 50, 936. (d) Fu, W.; Xu, F.; Fu, Y.; Xu, C.; Li, S.; Zou, D. Eur. J. Org. Chem. 2014, 709. (e) Wei, W.; Wen, J.; Yang, D.; Liu, X.; Guo, M.; Dong, R.; Wang, H. J. Org. Chem. 2014, 79, 4225. (f) Lu, Q.-Q.; Liu, C.; Peng, P.; Liu, Z.-L.; Fu, L.-J.; Huang, J.-G.; Lei, A.-W. Asian J. Org. Chem. 2014, 3, 273.

(22) Liu, J.-D.; Zhuang, S.-B.; Gui, Q.-W.; Chen, X.; Yang, Z.-Y.; Tan, Z. Eur. J. Org. Chem. 2014, 3196.

(23) Wang, J.-Y.; Zhang, X.; Bao, Y.; Xu, Y.-M.; Cheng, X.-F.; Wang, X.S. Org. Biomol. Chem. 2014, 12, 5582.
(24) (a) Kong, W.-Q.; Casimiro, M.; Merino, E.; Nevado, C. J. Am. Chem. Soc. 2013, 135, 14480. (b) Kong, W.-Q.; Merino, E.; Nevado, C. Angew. Chem. Int. Ed. 2014, 53, 5078. (c) Fuentes, N.; Kong, W.-Q.; Fernández-Sánchez, L.; Merino, E.; Nevado, C. J. Am. Chem. Soc. 2015, 137, 964.

(25) (a) Chan, C.-W.; Zhou, Z.; Chan, A. S. C.; Yu, W.-Y. Org. Lett. 2010, 12, 3296. (b) Jia, X.; Zhang, S.; Wang, W.; Luo, F.; Cheng, J. Org. Lett. 2009, 11, 3120. (c) Tang, B.-X.; Song, R.-J.; Li, J.-H. J. Am. Chem. Soc. 2010, 132, 8900. (d) Wu, Y.; Li, B.; Mao, F.; Li, X.; Kwong, F. Y. Org. Lett. 2011, 13, 3258.

(26) Zhou, M.-B.; Song, R.-J.; Ouyang, X.-H.; Liu, Y.; Wei, W.-T.; Deng, G.-B.; Li, J.-H. Chem. Sci. 2013, 4, 2690.

(27) Ouyang, X.-H.; Song, R.-J.; Li, J.-H. Eur. J. Org. Chem. 2014, 3395.

(28) Wang, H.; Guo, L.-N.; Duan, X.-H. Adv. Synth. Catal. 2013, 355, 2222.

(29) (a) Xu, X.; Tang, Y.; Li, X.; Hong, G.; Fang, M.; Du, X. J. Org. Chem. 2014, 79, 446. (b) Wang, G.; Wang, S.; Wang, J.; Chen, S.-Y.; Yu, X.-Q. Tetrahedron 2014, 70, 3466.

(30) Matcha, K.; Narayan, R.; Antonchick, A. P. Angew. Chem. Int. Ed. 2013, 52, 7985.

(31) (a) Wei, X.-H.; Li, Y.-M.; Zhou, A.-X.; Yang, T.-T.; Yang, S.-D. Org. Lett. 2013, 15, 4158. (b) Yuan, Y.; Shen, T.; Wang, K.; Jiao, N. Chem. Asian J. 2013, 8, 2932.

(32) Qiu, J.; Zhang, R.-H. Org. Biomol. Chem. 2014, 12, 4329.

(33) (a) Ono, N. The Nitro Group in Organic Synthesis; Wiley-VCH: Weinheim, 2001. (b) Feuer, H.; Nielson, A. T. Nitro Compounds: Recent Advances in Synthesis and Chemistry; VCH: Weinheim, 1990. (c) Barrett, A. G. M.; Graboski, G. G. Chem. Rev. 1986, 86, 751.

(34) (a) Li, Y.-M.; Wei, X.-H.; Li, X.-A.; Yang, S.-D. Chem. Commun. 2013, 49, 11701. (b) Li, Y.-M.; Shen, Y.; Chang, K.-J.; Yang, S.-D. Tetrahedron Lett. 2014, 55, 2119.

(35) Shen, T.; Yuan, Y.; Jiao, N. Chem. Commun. 2014, 50, 554.

(36) (a) Napier, C.; Stewart, M.; Melrose, H.; Hopkins, B.; McHarg, A.; Wallis, R. Eur. J. Pharmacol. 1999, 375, 61. (b) Petrov, K. G.; Zhang, Y.; Carter, M.; Cockerill, G. S.; Dickerson, S.; Gauthier, C. A.; Guo, Y.; Mook, R. A.; Rusnak, D. W.; Walker, A. L.; Wood, E. R.; Lackey, K. E. Bioorg. Med. Chem. Lett. 2006, 16, 4686.

(37) Li, X.; Xu, X.; Hu, P.; Xiao, X.; Zhou, C. J. Org. Chem. 2013, 78, 7343.

(38) Tian, Q.; He, P.; Kuang, C. Org. Biomol. Chem. 2014, 12, 6349.

(39) Wei, W.; Wen, J.; Yang, D.; Du, J.; You, J.; Wang, H. Green Chem. 2014, 16, 2988.

(40) Yin, F.; Wang, X.-S. Org. Lett. 2014, 16, 1128.

(41) (a) Van der Jeught, S.; Stevens, C. V. Chem. Rev. 2009, 109, 2672. (b) George, A.; Veis, A. Chem. Rev. 2008, 108, 4670. (c) Bialy, L.; Waldmann, H. Angew. Chem. Int. Ed. 2005, 44, 3814. (d) Alexandre, F.; Amador, A.; Bot, S.; Caillet, C.; Convard, T.; Jakubik, J.; Musiu, C.; Poddesu, B.; Vargiu, L.; Liuzzi, M.; Roland, A.; Seifer, M.; Standring, D.; Storer, R.; Dousson, C. B. J. Med. Chem. 2011, 54, 392.

(42) Li, Y.-M.; Sun, M.; Wang, H.-L.; Tian, Q.-P.; Yang, S.-D. Angew. Chem. Int. Ed. 2013, 52, 3972.

(43) Li, Y.-M.; Shen, Y.; Chang, K.-J.; Yang, S.-D. Tetrahedron 2014, 70, 1991. 